\title{
Proteção Renal na Unidade de Terapia Intensiva Cirúrgica*
}

\author{
Renal Protection in a Surgical Intensive Care Unit
}

\author{
Luciana Moraes dos Santos', Ludhmila Abrahão Hajjar², Filomena Regina Barbosa Galas³, \\ Constantino José Fernandes Júnior', José Otávio Costa Auler Júnior.
}

\section{RESUMO}

JUSTIFICATIVA E OBJETIVOS: A disfunção renal perioperatória é importante causa de aumento de morbimortalidade. Com o aumento da expectativa de vida, pacientes mais idosos e com maior números de co-morbidades estão sendo submetidos à procedimentos cirúrgicos de alto risco, o que torna as práticas da proteção orgânica possíveis modificadoras de prognóstico a curto e longo prazo. Nesse contexto, esta revisão sobre a proteção renal na unidade de terapia intensiva cirúrgica objetivou destacar os fatores de riscos peri-operatórios e discutir as atuais evidências científicas direcionadas para a diminuição da disfunção renal peri-operatória.

CONTEÚDO: Apesar da baixa extração e adequada reserva renal de oxigênio, o rim é extremamente sensível

1. Médica Assistente do Serviço de Anestesiologia e Terapia Intensiva Cirúrgica do InCor do HCFMUSP, Pós-Graduanda do Programa de Pós-Graduação em Anestesiologia da FMUSP.

2. Médica Assistente do Serviço de Anestesiologia e Terapia Intensiva Cirúrgica do InCor do HCFMUSP, Pós-Graduanda do Programa de Ciências Médicas da FMUSP. Especialista em Terapia Intensiva.

3. Médica Supervisora do Serviço de Anestesiologia e Terapia Intensiva Cirúrgica do InCor do HCFMUSP. Mestre em Anestesiologia pela FMUSP. Doutora em Ciências pela FMUSP. Especialista em Terapia Intensiva.

4. Gerente de Prática Médica do Hospital Israelita Albert Einstein. Secretário da Comissão de Título da Associação de Medicina Intensiva Brasileira

5. Professor Titular da Disciplina de Anestesiologia da FMUSP. Diretor do Serviço de Anestesiologia e Terapia Intensiva Cirúrgica do InCor do HCFMUSP.

*Recebido do Instituto do Coração, Hospital de Clínicas da Faculdade de Medicina da Universidade de São Paulo (HCFMUSP), São Paulo, SP

Apresentado em 11 de maio de 2006

Aceito para publicação em 22 de agosto de 2006

Endereço para correspondência:

Dra. Luciana Moraes dos Santos

Av. Dr. Arnaldo, $455-2^{\circ}$ andar

05430-100 São Paulo, SP

Fone (11) 3069-5232

E-mail: lumoraesrj@yahoo.com.br, luciana.santos@incor.usp.br

(C)Associação de Medicina Intensiva Brasileira, 2006 à hipoperfusão sendo a insuficiência renal aguda uma complicação freqüente de instabilidade hemodinâmica. Este aparente paradoxo, com alto suprimento de oxigênio e reduzida extração, com alta incidência de lesão renal à hipotensão, é explicado pelo gradiente fisiológico intra-renal de oxigênio que torna a medula particularmente susceptível à isquemia. Identificou-se fatores de lesão renal em todas as fases do período peri-operatório: jejum e uso de contraste pré-operatório, hipovolemia, hipotensão, liberação de catecolaminas e citocinas, utilização e circulação extracorpórea, politrauma, presença de rabdomiólise e pinçamento aórtico. É necessário que práticas capazes de diminuir a lesão renal peri-operatória sejam discutidas.

CONCLUSÕES: O controle da lesão renal baseia-se nos princípios da fisiologia renal peri-operatória e na otimização da hemodinâmica glomerular. Medidas direcionadas para proteção orgânica devem ser implementadas devido ao impacto da insuficiência renal na evolução clínica neste grupo de pacientes.

Unitermos: disfunção renal, insuficiência renal, peri-operatório, proteção renal.

\section{SUMMARY}

BACKGROUND AND OBJECTIVES: Perioperative renal dysfunction is an important cause of morbidity and mortality. With increase of life expectancy, older patients with more co-morbidity are being submitted to high risk surgical procedures, what make clinical practice related to organ protection possible modifier of short and long term survival. This review about renal protection in surgical intensive care unit points risk factors and discusses scientific evidence related to reduction of renal dysfunction in perioperative.

CONTENTS: Although low extraction and adequate renal reserve of oxygen, the kidney is extremely sensible to hypoperfusion being renal acute insufficiency a frequent complication of hemodynamic instability. This apparent paradox, high oxygen content and reduced extraction with high incidence of renal damage to hypotension re- 
flects the intra-renal gradient of oxygen, what makes renal medulla highly susceptible to ischemia. Factors associated with renal lesion are observed in all fases of perioperative period: fasting, contrast use, hypovolemia, hypotension, catecholamine and cytokine release, extracorporeal circulation, trauma, rabdomiolisys and aortic clamp.

CONCLUSIONS: Management of renal damage is based in principals of perioperative renal physiology and glomerular hemodynamic. Clinical practice directed to organic protection should be implemented to minimize the impact this dysfunction.

Key Words: perioperative, renal dysfunction, renal protection, renal insufficiency.

\section{INTRODUÇÃO}

Estudos demonstraram que o controle fisiológico e o suporte clínico adicional peri-operatório podem reduzir a mortalidade e morbidade no paciente cirúrgico ${ }^{1}$. A terapia intensiva cirúrgica atual se direciona para a proteção orgânica peri-operatória e com isso tem o objetivo de otimizar a evolução clínica do paciente, nesse contexto a proteção renal é essencial.

O período peri-operatório pode alterar a função renal de forma significativa e o surgimento da sua disfunção é importante causa de aumento de morbidade e mortalidade.

O entendimento da fisiologia renal peri-operatória é importante para o estudo dos mecanismos de lesão renal neste período e na aplicação das medidas protetoras.

O objetivo deste estudo foi discutir a fisiologia renal perioperatória, os mecanismos de lesão renal e as medidas utilizadas na proteção renal.

\section{DISFUNÇÃO RENAL PERI-OPERATÓRIA: DEFINIÇÃO E INCIDÊNCIA}

A disfunção renal peri-operatória é causa de aumento de morbimortalidade e pode ser considerada se houver aumento de $>0,5$ mg.dL ${ }^{-1}$ ou elevação em $50 \%$ do valor basal da creatinina ${ }^{2}$. Um estudo de 2672 pacientes submetidos à revascularização do miocárdio mostrou incidência de insuficiência renal aguda (IRA) pós-operatória de $8 \%$ com mortalidade de $14 \%{ }^{3}$. Outros estudos evidenciaram mortalidade de $60 \%$ a $90 \%$ relacionados à disfunção re$\mathrm{nal}^{4}$. Em pacientes submetidos à cirurgia cardíaca a IRA foi associada à maior tempo de UTI e de internação hospitalar. A IRA pós-operatória está relacionada a aumento do risco de infecção, sangramento gastrintestinal e sep$\mathrm{se}^{5}$. O maior preditor de disfunção renal pós-operatória em procedimentos de grande porte é a redução da função renal prévia à cirurgia.

\section{FISIOLOGIA RENAL PERI-OPERATÓRIA}

O rim recebe aproximadamente $20 \%$ do débito cardíaco com oferta total de oxigênio de $80 \mathrm{~mL} \cdot \mathrm{min}^{-1} .100 \mathrm{~g}^{-1}$ de tecido, sendo $90 \%$ direcionado para o córtex. O consumo de oxigênio renal não excede a 10\%, evidenciado por reduzida diferença arteriovenosa de oxigênio renal, ou seja, $1,5 \mathrm{~mL}$ de oxigênio por $100 \mathrm{~mL}$ de sangue. Apesar da baixa extração e adequada reserva renal de oxigênio, o rim é extremamente sensível a hipoperfusão, sendo a IRA uma complicação freqüente de instabilidade hemodinâmica. Este aparente paradoxo, alto suprimento de oxigênio e reduzida extração com alta incidência de lesão renal à hipotensão, é explicado pelo gradiente fisiológico intrarenal de oxigênio que torna a medula renal funcionante em ambiente hipóxico com 2-3 Kpa, devido ao maior consumo de oxigênio pelo transporte tubular renal de cloreto e sódio ${ }^{2}$. Embora $90 \%$ da oferta renal de oxigênio seja direcionado ao córtex, ou seja, $5 \mathrm{~mL} \cdot \mathrm{min}^{-1} \cdot \mathrm{g}^{-1}$, este extrai apenas $18 \%$, no entanto a medula, apesar de seu fluxo renal reduzido, $\left(0.03 \mathrm{~mL} \cdot \mathrm{min}^{-1} \cdot \mathrm{g}^{-1}\right)$ extrai $79 \%$.

A oxigenação medular renal é estritamente regulada por diversos fatores que controlam a relação entre a oferta e o consumo de oxigênio. A falha nessa regulação ocasiona lesão hipóxica com necrose tubular aguda (NTA) principalmente no ramo ascendente espesso ou em segmentos proximais 6 . A NTA pode ser induzida com uma redução de $40 \%$ a $50 \%$ no fluxo sanguíneo renal.

$\mathrm{O}$ trabalho associado à reabsorção de sal e água é fator predisponente à lesão medular hipóxica, o rim se beneficia de volume intravascular e carga de sal adequada, reduzindo a necessidade de concentração urinária.

O rim é desprovido de receptores $\beta-2$, assim, o aumento das catecolaminas circulantes causa vasoconstrição renal por receptores $\alpha-1$ e ativação do sistema renina angiotensina-aldosterona (SRAA), como resultado, apenas de taxa de filtração renal normal, a isquemia intramedular pode ocorrer, especialmente na região ascendente da alça de Henle onde as ATPases são sensíveis aos efeitos da isquemia. O aumento dos hormônios simpaticomiméticos leva à vasoconstrição cortical renal, como um mecanismo compensatório de redistribuição do fluxo sanguíneo para medula renal, causando isquemia. Como conseqüência, ocorre redução da reabsorção de sódio na alça ascendente, levando a retorno túbulo-glomerular via mácula densa com ativação do sistema renina angiotensina-aldosterona e constrição mesangial glomerular. 


\section{DISFUNÇÃO RENAL PERI-OPERATÓRIA}

Fisiopatologia

A IRA peri-operatória é multifatorial e pode resultar em azotemia pré-renal ou mais freqüentemente, NTA por lesão aos néfrons medulares hipóxicos. Os arteriopatas tem reduzida perfusão renal que aliada à redução da massa de néfrons funcionante com a idade, resulta em disfunção renal pré-operatória. O uso pré-operatório de inibidor da ECA aumenta a incidência de IRA pós-operatória por perda da capacidade do sistema renina-angiotensina de compensar reduções na perfusão renal ${ }^{7}$. No período perioperatório o volume circulante efetivo encontra-se diminuído devido ao jejum pré-operatório, perdas cirúrgicas e hipovolemia relativa ou absoluta pós-operatória. Na presença de baixo volume circulante fatores vasoconstritores, retenção neurohumoral de sal por estímulo do SRAA, sistema simpatoadrenal e ADH causam vasoconstrição renal, redução na taxa de filtração glomerular (TFG) e carga de solutos filtrada.

O sistema simpatoadrenal leva a aumento de adrenalina e noradrenalina direcionado para a arteríola aferente. A aldosterona aumenta a reabsorção de sal e água no túbulo coletor e proximal. No período peri-operatório devese evitar fármacos nefrotóxicos como antiinflamatórios não-esteróides, inibidores da enzima conversora de angiotensina ou antagonistas do receptor de angiotensina II porque a pressão hidrostática intraglomerular nesses pacientes é dependente da vasodilatação induzida por prostaglandinas, mantida pela arteríola aferente e angiotensina II e por vasoconstrição da arteríola eferente.
A disfunção renal pode agravar-se no pós-operatório se houver disfunção cardiovascular, sepse, ventilação com pressão positiva, exposição a fármacos nefrotóxicos. Os fatores relacionados à disfunção renal peri-operatória estão apresentados na tabela 1.

\section{Balanço de Fluidos Peri-Operatórios}

A reposição volêmica intra-operatória deve incluir: reposição da perda hídrica no jejum avaliada em $2 \mathrm{~mL} . \mathrm{kg}^{-1} . \mathrm{h}^{-}$ ${ }^{1}$, perdas insensíveis com a respiração e vasodilatação cutânea estimadas em 4-6 mL. $\mathrm{kg}^{-1} \cdot \mathrm{h}^{-1}$ e a perda cirúrgica que varia de acordo com o seu porte. Em procedimentos cirúrgicos de pequeno porte considera-se perda de 4 a 6 $\mathrm{mL} \cdot \mathrm{kg}^{-1} \cdot \mathrm{h}^{-1}$, de médio porte perdas de 6 a $10 \mathrm{~mL} \cdot \mathrm{kg}^{-1} \cdot \mathrm{h}^{-1}$ e de grande porte, perdas de 10 a $15 \mathrm{~mL} \cdot \mathrm{kg}^{-1} \cdot \mathrm{h}^{-1}$. O volume urinário deve ser considerado na reposição volêmica peri-operatória. Um paciente de $70 \mathrm{~kg}$, submetido à cirurgia de grande porte, com duração de 6 horas apresentará um déficit de volume de $9380 \mathrm{~mL}$ que devem ser repostos no período intra-operatório guiados por adequada monitorização. A estimativa adequada do volume de perda hídrica é essencial na prevenção de hipovolemia intra e pós-operatória. A hipovolemia impõe maior estresse reduzindo o suprimento de oxigênio renal ${ }^{9}$.

\section{Efeitos da Anestesia}

Tanto a anestesia regional quanto geral podem causar hipotensão e maior reposição de fluidos é necessária para manter a perfusão orgânica.

A anestesia leva à depressão miocárdica e vasodilatação

Tabela 1 - Disfunção Renal Peri-Operatória

\begin{tabular}{ll}
\hline Fatores Pré-Operatórios & Fatores Intra-Operatórios \\
\hline Idades > 70 anos & Circulação extracorpórea > 3 horas \\
Fração de ejeção menor que 35\% & Sangramento \\
Revascularização miocárdica & Liberação de citocinas (cirurgia, translocação) \\
Diabete melito tipo 2 & Complexo heparina-protamina (ativação de citocinas) \\
Hiperglicemia & Hipovolemia e hipotensão \\
Doença renal crônica & Vasodilatação induzida por anestésicos \\
Nefrosclerose hipertensiva & Disfunção miocárdica \\
Doença renal aterosclerótica & Aumento da pressão intra-abdominal (ascite) \\
Choque séptico & Ateroembolismo renal \\
Nefropatia por contraste (cateterismo cardíaco) & Embolização por fibrilação atrial aguda \\
Uso de antiinflamatórios não-esteróides (AINH) & Uropatia obstrutiva por agentes anestésicos \\
Aminoglicosídeos e anfotericina & Glomerulonefrite ou nefrite intersticial \\
Inibidores de calcineurina & Hipotermia \\
Uso de inibidores da ECA, $\beta$-agonistas e $\beta$-bloqueadores & Ventilação com pressão-positiva \\
Predisposição genética & Exposição a pigmentos biliares \\
& Rabdomiólise \\
\hline
\end{tabular}


o que exige reposição volêmica para manter a perfusão orgânica e a vasoconstrição da arteríola eferente, sendo esta responsável pela manutenção da pressão de filtração glomerular. O uso de narcóticos pode aumentar a liberação do $\mathrm{ADH}$ o que pode ser acentuado pelo jejum. O uso de ventilação mecânica exige uso de filtros humidificadores diminuindo os efeitos de gases secos utilizados.

\section{Efeitos da Cirurgia}

O procedimento cirúrgico leva a aumento de hormônios relacionados com a redução do volume urinário, entre eles o SRAA, aumento de ADH, glicocorticóides, citocinas e hormônios catabólicos.

A perda de fluidos pelo próprio procedimento cirúrgico é potencializada pelo estado de vasodilatação generalizada que se instala no período intra-operatório. Ocorre extravasamento de fluidos para o terceiro espaço decorrente de vasoplegia e neste período a reposição volêmica adequada deve ser mantida. Após a cirurgia o fluido se mobiliza de volta para o compartimento intravascular. A observação deste fenômeno ocorre principalmente em cirurgias intra-abdominais e intra-torácicas².

\section{CIRURGIA CARDÍACA}

\section{Circulação Extracorpórea}

A insuficiência renal aguda após circulação extracorpórea (CEC) ocorre entre $1 \%$ e $15 \%$ e está associada com mortalidade de $19 \%$. A incidência de IRA após revascularização do miocárdio com CEC que necessita de diálise é menor que $2 \%$ com mortalidade entre $23 \%$ e $88 \%^{2}$. A ocorrência de disfunção renal subclínica foi descrita e a preocupação existe em relação a possível capacidade do rim afetado subclinicamente ser submetido a nova lesão ${ }^{10}$. A lesão renal pela CEC é multifatorial, ocorre lesão por isquemia-reperfusão, presença de fluxo não pulsátil, micro e macroembolização renal, lesão por liberação traumática de hemoglobina e mioglobina na presença de isquemia muscular e rabdomiólise. A CEC impõe uma redução de 25\% a $75 \%$ do fluxo sanguíneo renal e aumento da resistência vascular sistêmica. Em condições normais, o fluxo sanguíneo renal mantém a medula em condição hipoxêmica, sendo estes néfrons de alta demanda e extração de oxigênio, sensíveis a modificações na oxigenação. Por isso o fluxo renal é mantido preferencialmente para manter a filtração em detrimento da função tubular, o que torna a medula renal susceptível à isquemia. Anteriormente, a lesão renal era atribuída somente à lesão tubular, no entanto, atualmente a influência da vasculatura intra-renal na redução do fluxo sanguíneo renal tem sido estudada ${ }^{11}$. A redução do fluxo sanguíneo renal é resistente ao aumento do débito cardíaco mesmo quando a taxa de filtração glomerular retorna ao normal, sugerindo que a isquemia renal ainda possa estar ocorrendo secundária à redução do fluxo regional. Isso ocorre por vasoconstrição renal intensa secundária à liberação de endotelina-1, acoplada à resposta exagerada a vasoconstritores e reduzida a vasodilatadores. A liberação de cálcio após isquemia causa aumento de endotelina. O rim evolui com edema e obstrução vascular o qual surge dentro e fora dos vasos renais co-existindo edema intersticial e de células epiteliais. Alterações descritas nos vasos renais:

- Redução do fluxo sanguíneo capilar;

- Disfunção endotelial;

- Up-regulation de moléculas de adesão e aumento de endotelina;

- Diminuição da produção de óxido nítrico e inibição da adesão.

Na tabela 2 estão descritos os mecanismos de lesão renal em cirurgia cardíaca.

Tabela 2 - Lesão Renal em Cirurgia Cardíaca

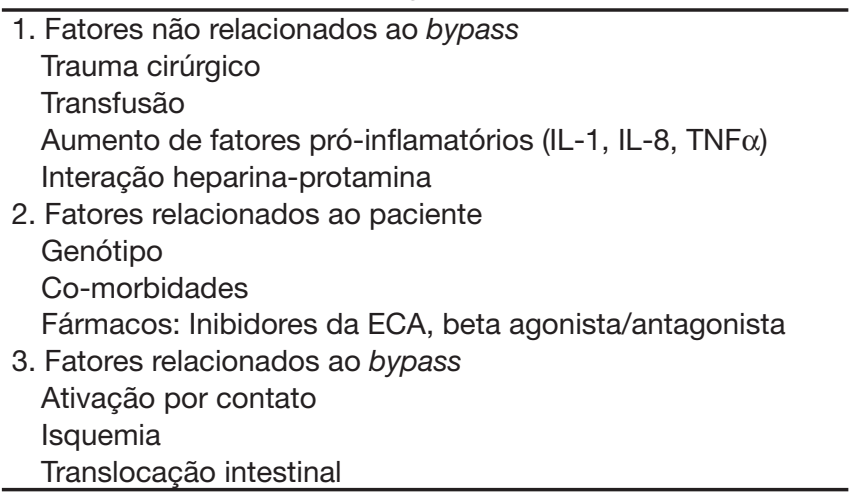

\section{Cirurgia de Aorta}

A incidência de necrose tubular aguda é significativa após cirurgias que envolvam as aortas torácica e abdominal supra-renal. Godet e col., descreveram incidência de disfunção renal peri-operatória de 20\% a 25\% em 475 pacientes submetidos à cirurgia de aorta ${ }^{12}$. Stevenson e col., relataram incidência de insuficiência renal aguda necessitando de diálise de 5,5\% e mortalidade hospitalar de $64 \%$. Neste estudo os principais fatores relacionados à IRA foram disfunção renal pré-existente, aterosclerose difusa, uso de CEC e instabilidade hemodinâmica.

A cirurgia de aorta é marcada por instabilidade hemodinâmica resultando em hipotensão e hipoperfusão renal 
decorrente da manipulação aórtica. A redução do fluxo sanguíneo renal após o pinçamento perdura por 48 horas sendo a redução $39 \%$ menor da observada no pinçamento infra-renal. A ausência de fluxo renal no pinçamento, embolia por manipulação da aorta e artéria renal e liberação de citocinas leva à isquemia visceral. A reperfusão após a liberação da pinça potencializa a lesão isquêmica renal. Quando mais alto o pinçamento mais vísceras estarão submetidas à lesão isquêmica e mais intensa é a resposta inflamatória com liberação de citocinas, potenciais causadoras de vasoconstrição e trombose da microvasculatura renal ${ }^{9}$.

O pinçamento infra-renal leva à redução do fluxo sanguíneo renal em torno de $40 \%$ como conseqüência do aumento da resistência vascular em até $75 \%$. A redução do volume urinário deve-se também à redução do débito cardíaco e do aumento de substâncias vasoconstritoras renais como a liberação de renina.

\section{Nefropatia por Contraste e Peri-Operatório}

O uso do contraste causa nefropatia por vasoconstrição intra-renal, redução do fluxo sanguíneo medular e ação osmótica levando a aumento da demanda medular de oxigênio ${ }^{4}$. O uso de contraste pode relacionar-se ao período peri-operatório em pacientes submetidos a cateterismo cardíaco previamente ao procedimento cirúrgico, ao surgimento de quadros isquêmicos peri-operatórios, em procedimentos tomográficos relacionados à doença cirúrgica e em procedimentos vasculares. A instituição de medidas direcionada para diminuir a agressão pelo contraste ao rim é importante aliada na redução da morbimortalidade peri-operatória.

Define-se nefropatia por contraste o aumento de 0,5 mg.dL${ }^{1}$ ou $25 \%$ da creatinina sérica após exposição a meio de contraste. Ela foi identificada por Nash e col., como a terceira causa mais comum de insuficiência renal aguda em pacientes hospitalizados, tendo sido considerada a nefropatia causada por infusão de contraste em 24 horas da piora renal sem outra evidência de lesão. A nefropatia por contraste soma-se à outros fatores de agressão renal no período peri-operatório como sangramento e hipovolemia, trauma cirúrgico, doença aterosclerótica e substâncias nefrotóxicas ${ }^{13}$ podendo ser causa de terapia dialítica. Estudos relatam incidência de 0,44\% de necessidade de diálise após intervenção coronariana com contraste ${ }^{14}$.

São descritos como fatores de risco para nefropatia por contraste: diabete melito, idade maior que 75 anos, hipovolemia, insuficiência cardíaca, cirrose, nefrose, hipertensão arterial, proteinúria, uso de antiinflamatório e inje- ção intra-arterial de contraste ${ }^{15}$. No contexto de isquemia coronariana e intervenção coronariana percutânea, hipotensão e uso de balão intra-aórtico foram associados à maior falência renal aguda ${ }^{16,17}$.

A nefropatia por contraste é geralmente temporária com pico de creatinina em três dias após a administração e retorno à linha de base em 10 dias $^{17}$.

\section{Transplante Renal e Peri-Operatório}

A necroso tubular aguda (NTA) é a causa mais comum de falência do enxerto com insuficiência renal aguda no período pós-operatório imediato e redução da sobrevida do enxerto ${ }^{18}$. Oligúria temporária ou anúria por NTA é mais comum após transplante de doador cadáver. O risco de necrose tubular aguda aumenta com o tempo de preservação do enxerto, uso de altas doses de ciclosporina, com o uso de vasopressor no doador e agentes nefrotóxicos. Causas cirúrgicas devem ser excluídas como sangramento, trombose arterial e venosa. Rejeição aguda deve ser considerada em caso de oligúria ${ }^{18}$. Reposição de fluidos guiada por pressão venosa central, pressão arterial e débito urinário é necessária para prevenir hipovolemia. Inotrópicos devem ser utilizados se a pressão sanguínea ou débito cardíaco estiverem inadequados.

\section{TESTES DE FUNÇÃO RENAL}

O ritmo de filtração glomerular (RFG) é, provavelmente, o mais importante indicador de disfunção renal. A determinação clássica da função renal pelo RFG necessita coleta de urina por 24 horas, no entanto as modificações na função renal ocorrem em menor período de tempo. A possibilidade de mensuração do RFG com depuração de duas horas foi investigada por Sladen e col. ${ }^{19}$. A estimativa do RFG na unidade de terapia intensiva ou pós-operatória é um indicador útil de alteração da função renal.

\section{Cistatina C}

Embora a medida do RFG seja útil, não é prática e medidas da creatinina sofrem influência de idade, sexo, massa muscular e dieta. Atualmente a dosagem de cistatina C tem sido investigada. É uma proteína alcalina não glicosilada produzida por todas as células nucleadas. No rim normal é totalmente filtrada e absorvida pela membrana glomerular e degradada por células proximais tubulares. Uma metanálise de Dharnidharka e col., compararam a cistatina $\mathrm{C}$ e a creatinina como indicadores do RFG e mostraram que a primeira foi superior e que 
refletem mais precocemente as suas alterações. Alterações de $50 \%$ do fluxo sanguíneo renal podem existir com valores normais de creatinina ${ }^{20}$. Os valores da cistatina $C$ são os mesmos para homens e mulheres sem influência da massa muscular, não sendo alterados em idosos.

RFG $\left(\mathrm{mL} \cdot \mathrm{min}^{-1}\right)=69.3 \times$ cistatina C $\left(\mathrm{mg}^{\mathrm{L}} \mathrm{L}^{-1}\right)$

Até o momento não existem estudos sobre o uso da cistatina $\mathrm{C}$ no pós-operatório.

\section{ESTRATÉGIAS DE PROTEÇÃO RENAL}

Grande parte da estratégia de proteção renal é baseada em tradição ou extrapolação de modelos animais visto que existem poucos estudos aleatórios. A manutenção de um débito urinário acima de $0,5 \mathrm{~mL} \cdot \mathrm{kg}^{-1} \cdot \mathrm{min}^{-1}$ é pratica comum, no entanto não existe evidência científica que suporte esse objetivo.

A incidência de insuficiência renal por todas as causas descritas, está relacionada com mortalidade de $69 \%$ a $90 \%$. Embora grande número de estratégias protetoras tenha sido proposto, nenhuma, exceto a manutenção de normovolemia pareceu ser efetiva. As demais terapias aguardam evidências científicas consistentes para serem definitivamente incluídas na prática médica. Estudos mostram que o manitol e a furosemida intra-operatórios estão associados à disfunção renal|21,22.

\section{Medidas Protetoras}

\section{Otimização da Perfusão Renal}

A adequação da perfusão renal é determinada pelo balanço entre o fluxo sanguíneo renal (FSR) e sua distribuição, e o consumo de oxigênio do parênquima. Os três determinantes principais do fluxo sanguíneo renal são o débito cardíaco, pressão de perfusão renal, hemodinâmica glomerular, ou seja, tônus das arteríolas eferentes e aferentes. Além disso, o consumo renal de oxigênio tem dois determinantes: a distribuição córtico-medular de fluxo e o consumo tubular de oxigênio com a reabsorção do sódio filtrado.

\section{Débito Cardíaco}

Os rins recebem $20 \%$ a $25 \%$ do débito cardíaco e constituem menos de $1 \%$ da massa corporal total sendo a maior relação-fluxo por tecido perfundido. A redução do débito cardíaco (DC) seja por hipovolemia ou disfunção miocárdica reduz a perfusão renal. A redução do débito cardíaco não apenas reduz diretamente o FSR mas inicia a produção de vasoconstritores por ativação do
SRAA, sistema nervoso simpático e vasopressina, tendendo a aumento da pressão de perfusão e vasoconstrição renal. O aumento do DC e a perfusão sistêmica são essenciais para melhorar a perfusão renal e por isso atenção ao volume circulante prevenindo hipovolemia é essencial para prevenir e diminuir a disfunção renal perioperatória. O uso de inotrópicos deve ser instituído, se necessário, na prevenção do baixo débito peri-operatório enquanto a disfunção renal estiver presente.

Reposição Volêmica - Colóides Versus Cristalóides na Disfunção Renal

Os cristalóides podem ser administrados para manter a perfusão renal, no entanto, em pacientes com hipovolemia relativa e aumento de permeabilidade vascular devido à inflamação, os colóides devem ser utilizados. Kumle e col., demonstraram que o uso dos hydroxyethyl starches (HES) de baixo peso molecular na disfunção renal peri-operatória está associado com aumento de indicadores específicos de lesão tubular renal como alfa1-microglobulina, no entanto esta elevação pode estar relacionada com o trauma cirúrgico propriamente dito e não com a utilização da solução ${ }^{23}$. Em pacientes críticos o uso de HES não esteve relacionado à aumento de creatinina, oligúria e progressão para diálise ${ }^{24}$. Estudos mostraram que o HES de médio e alto peso podem estar relacionados com disfunção renal em pacientes cirúrgicos e sépticos ${ }^{25,26}$. Jungheinrich e col. demonstraram em voluntários com disfunção renal estável, não anúrica que o HES de baixo peso pode ser administrado, pois não há acúmulo plasmático e ocorre preservação do fluxo urinário ${ }^{27}$. Em relação à albumina, estudos mostraram efeito protetor renal com redução da incidência de disfunção renal de $33 \%$ para $10 \%(p<0,02)$ e redução da mortalidade hospitalar de $29 \%$ para $10 \%(p<0,01)$ em estudo aleatório com 126 pacientes com peritonite bacteriana $^{28}$. As diferentes propriedades da albumina e colóides semi-sintéticos devem ser consideradas na reposição volêmica do paciente renal.

\section{Pressão de Perfusão Renal}

A auto-regulação renal mantém o FSR e RFG com pressão arterial média (PAM) entre 85 e $180 \mathrm{mmHg}$. No entanto a PAM e a pressão de perfusão renal-alvo para suporte hemodinâmico adequado é incerta. A auto-regulação renal é alterada em pacientes hipertensos crônicos, na sepse e na circulação extracorpórea. O limite clássico de 60 $\mathrm{mmHg}$ está no limite inferior de perfusão dos principais órgãos. Em estudo aleatório, foi utilizada noradrenalina em pacientes sépticos para PAM de 65, 75 e 85 mmHg. 
As variáveis hemodinâmicas, índices metabólicos, perfusão esplâncnica, perfusão cutânea e débito urinário foram monitorizados. Não houve diferença significativa entre o lactato arterial, a saturação venosa mista e os índices de perfusão orgânica entre os grupos. Índice cardíaco e resistência vascular sistêmica aumentaram com a dose de noradrenalina ${ }^{29}$. Os estudos que obtiveram os mesmos resultados demonstraram que a PAM de $65 \mathrm{mmHg}$ é um limite razoável, no entanto, a individualização é necessária, tanto para valores inferiores guiados por índices de perfusão tecidual quanto para valores superiores nos pacientes com doença vascular ${ }^{30}$.

\section{Otimização da Hemodinâmica Glomerular}

A vasoconstrição renal no período peri-operatório é conseqüência de hipovolemia, aumento de citocinas, baixo débito cardíaco, sepse e síndrome hepato-renal. O real impacto dessa vasoconstrição renal no período peri-operatório ainda não foi definido, no entanto, teoricamente qualquer agente que diminua a vasoconstrição renal seria capaz de reduzir a incidência de IRA pré-renal e NTA em pacientes de alto risco. A utilização de vasodilatadores no período peri-operatório deve ser criteriosa, nas primeiras horas após a cirurgia e no paciente ainda sob os efeitos deste período, devendo-se optar pela escolha de vasodilatadores venosos como nitroprussiato de sódio. A utilização de vasodilatadores orais de duração mais prolongada exige estabilidade hemodinâmica e ausência de processo infeccioso. O uso de fenoldopam tem sido estudado na disfunção renal de pacientes críticos (Tabelas 3 e 4).

Tabela 3 - Risco de Disfunção Renal Aguda após Intervenção Coronariana Percutânea ${ }^{16}$

\begin{tabular}{|c|c|}
\hline Fator de Risco & Escore \\
\hline $\begin{array}{l}\text { Pressão sistólica }<80 \text { mmHg } \\
\text { e uso de inotrópico ou balão } \\
\text { intra-aórtico }(\mathrm{BIA}) \text { em } 24 \text { horas } \\
\text { após o procedimento }\end{array}$ & 5 \\
\hline Uso de BIA & 5 \\
\hline $\begin{array}{l}\text { ICC (NYHA III ou IV) e/ou histó- } \\
\text { ria de edema pulmonar }\end{array}$ & 5 \\
\hline Idade $>75$ anos & 5 \\
\hline $\begin{array}{l}\text { Hematócrito > } 39 \% \text { em ho- } \\
\text { mens e } 36 \% \text { em mulheres }\end{array}$ & 3 \\
\hline Diabete & 3 \\
\hline Volume de contraste & 1 para cada $100 \mathrm{~mL}$ \\
\hline $\begin{array}{l}\text { Creatinina }>1,5 \text { ou } \mathrm{RFG}^{*}<60 \\
\mathrm{~mL} \cdot \mathrm{min}^{-1} / 1,73 \mathrm{~m}^{2}\end{array}$ & 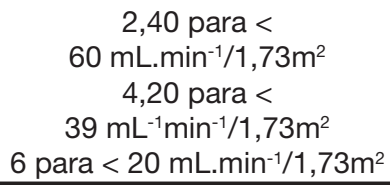 \\
\hline
\end{tabular}

*Estimado por Cockcroft-Gault
Tabela 4 - Valor do Escore e Risco de Diálise

\begin{tabular}{lcc}
\hline Escore & Risco & Risco de Diálise \\
\hline$<=5$ & 7,5 & 0,04 \\
$6-10$ & 14 & 0,12 \\
$11-15$ & 26,1 & 1,09 \\
$>=16$ & 57,3 & 12,6 \\
\hline
\end{tabular}

Nefropatia por Contraste

A prevenção da nefropatia por contraste inicia-se na estimativa do seu risco, é proposto um escore para quantificação deste risco.

\section{REPOSIÇÃO DE FLUIDOS: SOLUÇÃO FISIOLÓGICA VERSUS BICARBONATO ISOTÔNICO}

A hidratação é recomendada para prevenir o risco de nefropatia induzida por contraste, no entanto o regime ideal ainda não foi definido. Estudos recentes compararam a administração oral de fluidos com infusão venosa de solução fisiológica a $0,9 \%$ ou $0,45 \%$ e comprovaram a sua superioridade ${ }^{31,32}$. Mueller e col. demonstraram em 1620 pacientes que a solução fisiológica isotônica foi superior à hipotônica na prevenção da nefropatia por contraste ${ }^{33}$. A infusão dessa solução é recomendada previamente à administração de contraste. Até o momento em um único estudo aleatório, Merten e col., demonstraram que a infusão de solução isotônica de bicarbonato de sódio a 154 $\mathrm{mmol} / \mathrm{L}$ (bicarbonato de sódio a 8,4\% $150 \mathrm{~mL}$ em SF a $0,9 \% 850 \mathrm{~mL}$ ) na dose de $3 \mathrm{~mL} . \mathrm{kg}^{-1}$, uma hora antes da infusão de contraste e $1 \mathrm{~mL} \cdot \mathrm{kg}^{-1} \cdot \mathrm{h}^{-1}$ por seis horas foi capaz de reduzir significativamente a nefropatia induzida por contraste. Essa redução pode ser explicada pelo efeito da alcalinização em diminuir a formação de radicais livres gerada pelo contato do contraste com os túbulos renais, a qual é facilitada em ambientes ácidos ${ }^{34}$.

\section{N-acetilcisteína}

A N-acetilcisteína por ser substância vasodilatadora e antioxidante poderia diminuir a nefropatia por contraste, duas metanálises recentes demonstraram possíveis benéficos da sua administração, no entanto, os estudos incluídos foram heterogêneos e faltam evidências sobre os efeitos da $\mathrm{N}$-acetilcisteína na evolução clínica. A dose utilizada foi de $600 \mathrm{mg}$ via oral a cada 12 horas num total de 4 doses, no entanto dados mais consistentes são necessários para a recomendação do seu uso. 


\section{Escolha do Meio de Contraste}

Atualmente recomenda-se a utilização de contraste de baixa osmolaridade porque estudos mostraram que estes estão associados à menor nefrotoxicidade ${ }^{35}$. Além disso, recomenda-se que se utilize a menor dose possível visto que doses maiores que $5 \mathrm{~mL} . \mathrm{kg}^{-1}$ divididos pela creatinina plasmática em mg.dL-1 estão associados à maior risco (Tabela 5) ${ }^{14}$.

Tabela 5 - Recomendações Sumárias para Redução do Risco de Nefropatia por Contraste ${ }^{15}$

\begin{tabular}{|c|c|c|}
\hline Intervenção & Dose Estudada & Recomendação \\
\hline Hidratação venosa & $\begin{array}{l}\text { Solução fisiológica } \\
\text { a } 0,9 \% 1 \mathrm{~mL} \cdot \mathrm{kg}^{-1} \cdot \mathrm{h}^{-1} \\
\text { por } 24 \text { horas antes }\end{array}$ & Recomendada \\
\hline $\begin{array}{l}\text { Bicarbonato de Só- } \\
\text { dio } \\
154 \mathrm{mmol} / \mathrm{L}\end{array}$ & $\begin{array}{l}3 \mathrm{~mL} \cdot \mathrm{kg}^{-1} \cdot \mathrm{h}^{-1} 1 \text { hora } \\
\text { antes e } 3 \mathrm{~mL} \cdot \mathrm{kg}^{-1} \cdot \mathrm{h}^{-1} \\
\text { por } 6 \text { horas após }\end{array}$ & $\begin{array}{l}\text { Não recomendada } \\
\text { de rotina. Aguar- } \\
\text { dar evidência }\end{array}$ \\
\hline Meio de contraste & $\begin{array}{l}\text { Meio de baixa osmo- } \\
\text { laridade } \\
\text { Dose menor que } 5 \\
\mathrm{~mL} . \mathrm{kg}^{-1} / \text { creatinina }\end{array}$ & Recomendado \\
\hline N-acetilcisteína & $\begin{array}{l}600 \mathrm{mg} \text { via por oral } \\
12 / 12 \mathrm{~h} \text { iniciada antes } \\
\text { até } 4 \text { doses }\end{array}$ & $\begin{array}{l}\text { Não recomendada } \\
\text { de rotina. Aguar- } \\
\text { dar evidência }\end{array}$ \\
\hline
\end{tabular}

Não existem estudos sobre a utilização da solução de bicarbonato de sódio no contexto peri-operatório, no entanto os pacientes neste cenário possuem múltiplos fatores de agressão renal e maior potencial de complicação podendo beneficiar-se dessa solução, caso seja necessária a utilização de contraste.

\section{Insuficiência Renal Mioglobinúrica}

A rabdomiólise é uma entidade clínica que se caracteriza por lesão da musculatura esquelética. O período perioperatório pode cursar com rabdomiólise secundária à lesão muscular por trombose ou embolização na vasculatura periférica, pelo uso de balão intra-aórtico, má perfusão periférica, circulação extracorpórea, trauma cirúrgico e síndrome compartimental. A detecção dessas complicações pode exigir fasciotomia ou mesmo se manifestar pela presença de urina escura. A dosagem de creatinaquinase (CPK) é essencial na avaliação de possível lesão renal.

Associada à hipovolemia, a mioglobina liberada na circulação causa lesão renal por citotoxicidade direta, obstrução tubular e vasoconstrição renal. O nível de CK associado com lesão renal não foi definido, tendo sido descrito com níveis de $500 \mathrm{UI} / \mathrm{L}$ até $75000 \mathrm{UI} / \mathrm{L}$. Três estratégias bem definidas são hidratação venosa, manutenção da perfusão renal e débito urinário, para evitar obstrução tubular e alcalinização da urina com bicarbonato. No entanto o uso do bicarbonato e manitol são baseados em pequenos estudos com número reduzido de pacientes. Em estudo com pacientes críticos pós-trauma, os valores de CK acima de $5000 \mathrm{UI} / \mathrm{L}$ estiveram associados à maior lesão renal como fator de risco independente. Neste grupo de alto risco, para disfunção renal, a administração de bicarbonato de sódio e manitol não demonstrou diferença em relação à prevenção de insuficiência renal, diálise e mortalidade ${ }^{36}$.

Recomenda-se em pacientes com CK > $5000 \mathrm{UI} / \mathrm{L}$ a mensuração do pH urinário a cada 6 horas com administração de bicarbonato $\left(1 \mathrm{~mL} \cdot \mathrm{kg}^{-1}\right)$ em bolus objetivando a manutenção do $\mathrm{pH}$ urinário acima de 8.0 para alcalinização urinária e redução da precipitação intratubular de mioglobina. A dose ideal de bicarbonato a ser utilizada e o real impacto dessas medidas no desfecho clínico ainda está para serem definidos.

Otimização Farmacológica no Paciente com Disfunção Renal

No ambiente de terapia intensiva cirúrgica atenção especial deve ser direcionada aos fármacos utilizados no paciente com disfunção renal. Deve-se evitar administração de medicações que reduzam a pressão de perfusão glomerular como inibidores da enzima conversora e uso de antiinflamatórios não-esteróides. Os antimicrobianos devem ter suas doses corrigidas e monitorizadas quando possível. Em pacientes transplantados, atenção especial deve ser direcionada a ciclosporina por seu efeito nefrotóxico bem estabelecido.

\section{Controvérsias em Proteção Renal}

Diversas estratégias foram estudadas em renoproteção, como diuréticos, manitol, aminofilina, dopamina, dopexamina, no entanto nenhum estudo aleatório demonstrou real redução de insuficiência renal aguda.

\section{RECOMENDAÇÕES}

A normovolemia é medida essencial na proteção renal peri-operatória e a única comprovadamente eficaz². Observada como medida aparentemente simples, frente a tantas teoricamente sofisticadas estratégias de renoproteção, a manutenção da normovolemia exige complexa avaliação e monitorização. No período pós-operatório ob- 


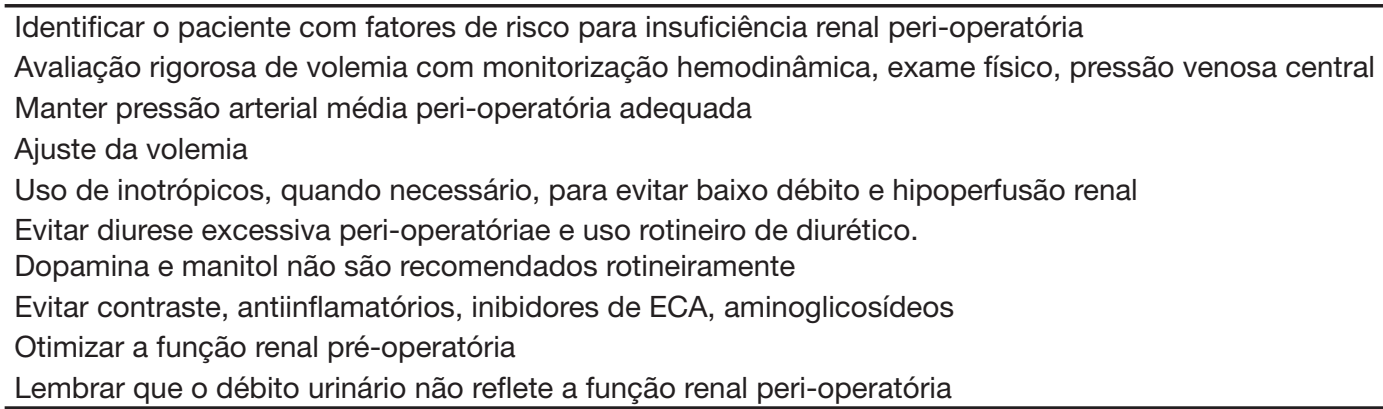

serva-se piora progressiva da função renal secundária à lesão cirúrgica e potencializada em certas situações pela administração prévia de contraste. Nesse contexto sugere-se a otimização monitorizada da volemia com balanço hídrico rigoroso, mensuração diária da função renal, peso e diurese, correção do déficit de base, manutenção de inotrópicos em baixas doses, racionalização na escolha da solução ideal para hidratação. A decisão de instituição de terapia de substituição renal deve ser individualizada, observando-se os riscos e os benefícios da técnica e o prognóstico da lesão renal. A utilização de diuréticos e manitol no período peri-operatório deve ser criteriosa devido ao risco de instalação de hipovolemia e piora da lesão renal. Apenas quando houver otimização monitorizada da volemia com medida de pressões cardíacas de enchimento, índices de perfusão global como excesso de base, lactato, gradiente venoarterial de dióxido de carbono $\left(\right.$ DeltaCO $\left.{ }_{2}\right)$ e saturação venosa de oxigênio, não sugestivos de hipovolemia, e/ou edema pulmonar clinicamente significativo pode-se administrar esses fármacos. O período peri-operatório cursa com hipovolemia relativa devido ao aumento da permeabilidade capilar que ocorre secundária ao trauma cirúrgico, por isso a administração de diuréticos deve ser criteriosa (Quadro 1).

\section{PERSPECTIVAS EM PROTEÇÃO RENAL}

\section{Fenoldopam}

O mesilato de fenoldopam é um vasodilatador renal, agonista seletivo do receptor dopamina-1 que aumenta os fluxos sanguíneos cortical e medular, reduzindo a resistência vascular sistêmica e teoricamente poderia melhorar a hemodinâmica glomerular. Diversos estudos têm surgido para determinar possíveis benefícios deste fármaco no peri-operatório e no paciente séptico, freqüentemente encontrado na unidade de terapia intensiva cirúrgica, no entanto, os resultados ainda são conflitantes. A infusão de fenoldopam foi estudada em 80 pacientes submetidos à cirurgia cardíaca com risco para disfunção renal perioperatória, numa dose de $0,05 \mu \mathrm{g} \cdot \mathrm{kg}^{-1} \cdot \mathrm{min}^{-1}$ até 24 horas de pós-operatório, comparado a infusão continua de 2,5 $\mu \mathrm{g} \cdot \mathrm{kg}^{-1} \cdot \mathrm{min}^{-1}$ de dopamina no mesmo período. Não se observou diferença na incidência de insuficiência renal (42\% versus $40 \%, p<0,9)$, pico de creatinina, internação na unidade de terapia intensiva e hospitalar e mortalidade entre os grupos. No entanto, este estudo comparou fenoldopam com dopamina e não com placebo e a dose utilizada foi maior do que a inicialmente dita como benéfica para a melhora da hemodinâmica glomerular. Embora este estudo tenha excluído o fenoldopam como estratégia possível na prevenção da lesão renal, as limitações existem e talvez tenham impedido a detecção de possíveis benefícios. Morelli e col. demonstraram em 300 pacientes sépticos que a infusão de fenoldopam contínuo $\left(0,09 \mu \mathrm{g} \cdot \mathrm{kg}^{-1} \cdot \mathrm{min}^{-1}\right)$ comparado com placebo reduziu significativamente $(p<0,006)$ o aumento da creatinina sérica e tempo de internação na unidade de terapia intensiva ( $p$ $<0,01)$ no entanto não evitou a disfunção renal grave $e$ não reduziu a mortalidade ${ }^{37}$.

\section{Agonistas do Receptor de Adenosina}

Estudos mostraram que esta classe de fármacos tem efeitos benéficos em modelos animais de lesão renal isquêmica ${ }^{38}$. Os agonistas de adenosina aumentam a perfusão da medula renal, região mais susceptível à isquemia. No entanto, nenhum estudo foi realizado em seres humanos até o momento.

\section{Bloqueadores da Resposta Inflamatória}

A prevenção da formação de radicais livres de oxigênio derivados de neutrófilos foi estudada na renoproteção. Winjen e col., demonstraram que a utilização de cinco antioxidantes reduziu a incidência de IRA em pacientes submetidos à correção de aneurisma de aorta abdominal ${ }^{39}$. 


\section{Aprotinina}

Mangano e col. demonstraram em estudo prospectivo e aleatório o aumento em duas vezes no risco de falência renal em 4375 pacientes submetidos à revascularização do miocárdio complexa (odds ratio 2,59; IC 95\%:1.36 à 4.95) e à primeira cirurgia (odds ratio de 2.34; IC 95\%: 1.27 à 4.31$)^{40}$. Nos grupos que receberam o ácido epsilon-aminocapróico e ácido tranexâmico não houve associação com eventos renais.

\section{CONCLUSÃO}

A unidade de terapia intensiva tem importante papel na redução de morbimortalidade do paciente cirúrgico. Medidas direcionadas para proteção orgânica devem ser implementadas. Devido ao impacto da insuficiência renal na evolução clínica deste grupo de pacientes a renoproteção é um aspecto essencial. Os efeitos de diversos fármacos na proteção renal têm sido estudados nos últimos anos, no entanto existem poucos estudos e os resultados são controversos. Atualmente a renoproteção baseia-se em otimização da volemia, da hemodinâmica glomerular e da diminuição de fatores que possam contribuir para a disfunção renal peri-operatória.

\section{REFERÊNCIAS}

01. Goldhill D - Intensive and high-dependency care for the high-risk surgical patient. Baillière's Clinical Anaesthesiology, 1999;13:427-436.

02. Sear JW - Kidney dysfunction in the postoperative period. $\mathrm{Br} \mathrm{J}$ Anaesth, 2005;95:20-32.

03. Conlon PJ, Stafford-Smith M, White WD et al - Acute renal failure following cardiac surgery. Nephrol Dial Transplant, 1999;14:1158-1162.

04. Levy EM, Viscoli CM, Horwitz RI - The effect of acute renal failure on mortality. A cohort analysis. JAMA, 1996;275:1489-1494.

05. Aronson S, Blumenthal R - Perioperative renal dysfunction and cardiovascular anesthesia: concerns and controversies. J Cardiothorac Vasc Anesth, 1998;12:567-586.

06. Brezis M, Rosen S - Hypoxia of the renal medulla - its implications for disease. N Engl J Med, 1995;332: 647-655.

07. Cittanova ML, Zubicki A, Savu C et al - The chronic inhibition of angiotensin-converting enzyme impairs postoperative renal function. Anesth Analg, 2001;93:1111-1115.

08. Tang IY, Murray PT - Prevention of perioperative acute renal failure: what works? Best Pract Res Clin Anaesthesiol, 2004;18:91-111.

09. Swaminathan $M$, Stafford-Smith $M$ - Renal dysfunction after vascular surgery. Curr Opin Anesthesiology, 2003;16:45-51.

10. Jorres A, Kordonouri O, Schiessler A et al - Urinary excretion of thromboxane and markers for renal injury in patients undergoing cardiopulmonary bypass. Artif Organs, 1994;18:565-569.

11. Lieberthal W, McKenney JB, Kiefer CR et al - Beta1 integrin-mediated adhesion between renal tubular cells after anoxic injury. J Am Soc Nephrol, 1997;8:175-183.

12. Godet $\mathrm{G}$, Fleron $\mathrm{MH}$, Vicaut $\mathrm{E}$ et al - Risk factors for acute postoperative renal failure in thoracic or thoracoabdominal aortic surgery: a prospective study. Anesth Analg, 1997;85:1227-1232.

13. Nash K, Hafeez A, Hou S - Hospital-acquired renal insufficiency. Am J Kidney Dis, 2002;39:930-936.

14. Freeman RV, O'Donnell M, Share D et al - Nephropathy requiring dialysis after percutaneous coronary intervention and the critical role of an adjusted contrast dose. Am J Cardiol, 2002;90:1068-1073.

15. Barrett BJ, Parfrey PS - Clinical practice. Preventing nephropathy induced by contrast medium. N Engl J Med, 2006;354:379-386.

16. Mehran R, Aymong ED, Nikolsky E et al - A simple risk score for prediction of contrast-induced nephropathy after percutaneous coronary intervention: development and initial validation. J Am Coll Cardiol, 2004;44:1393-1399.

17. Marenzi G, Lauri G, Assanelli E et al - Contrast-induced nephropathy in patients undergoing primary angioplasty for acute myocardial infarction. J Am Coll Cardiol, 2004;44:1780-1785.

18. Visser W - Anesthesia for renal transplantation. Curr Anesth and Crit Care, 1999;10:286-290.

19. Sladen RN, Endo E, Harrison T - Two-hour versus 22-hour creatinine clearance in critically ill patients. Anesthesiology, 1987;67:1013-1016.

20. Dharnidharka VR, Kwon C, Stevens G - Serum cystatin C is superior to serum creatinine as a marker of kidney function: a meta-analysis. Am J Kidney Dis, 2002;40:221-226.

21. Visweswaran P, Massin EK, Dubose TD - Mannitol-induced acute renal failure. J Am Soc Nephrol, 1997;8:1028-1033.

22. Kellum JA - The use of diuretics and dopamine in acute renal failure: a systematic review of the evidence. Crit Care, 1997;1:53-59.

23. Kumle B, Boldt J, Piper S et al - The influence of different intravascular volume replacement regimens on renal function in the elderly. Anesth Analg, 1999;89:1124-1130.

24. Yasser SRK, Payen D, Reinhart K, Fraintpont V, Gerard I, Vincent J L Effects of hydroxyetyl starch administration on renal function in critically ill patients. Crit Care Med, 2006;33(S):A175

25. Dehne MG, Muhling J, Sablotzki A et al - Effect of hydroxyethyl starch solution on kidney function in surgical intensive care patients. Anasthesiol Intensivmed Notfallmed Schmerzther, 1997;32:348-354.

26. Schortgen F, Lacherade JC, Bruneel F et al - Effects of hydroxyethylstarch and gelatin on renal function in severe sepsis: a multicentre randomised study. Lancet, 2001;357:911-916.

27. Jungheinrich $C$, Scharpf $R$, Wargenau $M$ et al - The pharmacokinetics and tolerability of an intravenous infusion of the new hydroxyethyl starch $130 / 0.4(6 \%, 500 \mathrm{~mL})$ in mild-to-severe renal impairment. Anesth Analg, 2002;95:544-551.

28. Sort P, Navasa M, Arroyo V et al - Effect of intravenous albumin on renal impairment and mortality in patients with cirrhosis and spontaneous bacterial peritonitis. N Engl J Med, 1999;341:403-409.

29. LeDoux D, Astiz ME, Carpati CM et al - Effects of perfusion pressure on tissue perfusion in septic shock. Crit Care Med, 2000;28:2729-2732.

30. Reisbeck M, Astiz ME - Arterial pressure, vasopressors and septic shock higher is not necessarily better. Crit Care Med, 2005;33:906-907.

31. Trivedi HS, Moore $\mathrm{H}, \mathrm{Nasr} \mathrm{S}$ et al - A randomized prospective trial to assess the role of saline hydration on the development of contrast nephrotoxicity. Nephron Clin Pract, 2003;93:C29-C34.

32. Bader BD, Berger ED, Heede MB et al - What is the best hydration regimen to prevent contrast media-induced nephrotoxicity? Clin Nephrol, 2004;62:1-7.

33. Mueller C, Buerkle G, Buettner HJ et al - Prevention of contrast mediaassociated nephropathy: randomized comparison of 2 hydration regimens in 1620 patients undergoing coronary angioplasty. Arch Intern Med, 2002;162:329-336.

34. Merten GJ, Burgess WP, Gray LV et al - Prevention of contrast-induced nephropathy with sodium bicarbonate: a randomized controlled trial. JAMA 2004;291:2328-2334.

35. Barrett BJ, Carlisle EJ - Meta-analysis of the relative nephrotoxicity of highand low-osmolality iodinated contrast media. Radiology, 1993;188:171-178.

36. Brown CV, Rhee P, Chan L et al - Preventing renal failure in patients with rhabdomyolysis: do bicarbonate and mannitol make a difference? J Trauma, 2004;56:1191-1196.

37. Morelli A, Ricci Z, Bellomo R et al - Prophylactic fenoldopam for renal protection in sepsis: a randomized, double-blind, placebo-controlled pilot trial. Crit Care Med, 2005;33:2451-2466.

38. Lee HT, Emala CW - Systemic adenosine given after ischemia protects renal function via $\mathrm{A}(2 \mathrm{a})$ adenosine receptor activation. Am J Kidney Dis, 2001;38:610-618.

39. Wijnen $\mathrm{MH}$, Roumen RM, Vader $\mathrm{HL}$ et al - A multiantioxidant supplementation reduces damage from ischaemia reperfusion in patients after lower torso ischaemia. A randomised trial. Eur J Vasc Endovasc Surg, 2002;23:486-490.

40. Mangano DT, Tudor IC, Dietzel C - The risk associated with aprotinin in cardiac surgery. N Engl J Med, 2006;354:353-365. 